\title{
Macroporous silicon: efficient antireflective layer on crystalline silicon
}

\author{
Faruk Fonthal $\cdot$ Ivaldo Torres $・$ Angel Rodriguez
}

Received: 16 April 2010/ Accepted: 6 October 2010

(C) Springer Science+Business Media, LLC 2010

\begin{abstract}
A macroporous silicon layer (ma-PS) electrochemically grown on crystalline silicon surface can be used as an efficient antireflective layer in optical devices as antireflection coating. In this work, we presented the maPS layers fabricated on crystalline silicon (c-Si) $n$-type and $p^{+}$-type, obtained by electrochemical etching. The morphology, porosity, thickness of ma-PS layer can be adjusted by controlling the electrochemical formation conditions. The optical behaviour of the antireflective coating over the solar spectrum is determined, resulting in very low values of the normalized reflectivity coefficient (below $\sim 1 \%$ ). The reflectivity measurements were evaluated at $45^{\circ}$ in the different samples of the ma-PS/c-Si.
\end{abstract}

\section{Introduction}

The first application of photo-electrochemically formed porous silicon (oxide) antireflection coatings (ARC) on

\footnotetext{
F. Fonthal $(\bowtie) \cdot$ I. Torres

Advanced Materials for Micro and Nanotechnology Group, IMAMNT, Departamento de Automática y Electrónica, Universidad Autónoma de Occidente, Calle 25 No. 115-85, Cali, Colombia

e-mail: ffonthal@uao.edu.co

I. Torres

e-mail: ivaldo.torres@unipamplona.edu.co

F. Fonthal · I. Torres

LOGOS Group, Departamento de IEEST, Universidad de

Pamplona, Km. 1 Vía a Bucaramanga s/n, Pamplona, Colombia

\author{
A. Rodriguez \\ Micro and Nanotechnology Group, Departament d'Enginyeria \\ Electrònica, Universitat Politècnica de Catalunya, C/Gran Capità \\ s/n, Campus Nord, 08074 Barcelona, Spain \\ e-mail: arm@eel.upc.edu
}

polycrystalline Si solar cells was reported in 1981 [1]. This defines the starting point in the search for a simple porous etch to obtain an ARC and a selective emitter structure, where they have been obtained low values of normalized reflectivity below 10\% [1]. Then the discovery of the photoluminescence porous silicon (PS) at roomtemperature in 1990 [2], have been publishing many work about this interesting material until today.

Macroporous silicon (ma-PS) has continued drawing the attention in the last decade because of its potential for creating textured antireflection coatings of silicon solar cells $[3,4]$ and for integrating optoelectronic and microelectronic elements based on silicon technology $[5,6]$. The ma-PS layer is formed by electrochemical etching with aqueous hydrofluoric (HF) solution on the crystalline silicon (c-Si) surface [7]. The electrochemical HF etching process on the c-Si wafer is still the most widely applied porous silicon formation techniques [3-7].

The advances in optic devices incorporate surface texturization to reduce the reflection and consequently improve the optic absorption. For this reason, PS is use as antireflective coating for solar cells or photodiodes, nowadays [8-12]. PS optical layers have drawn a lot of interest because of their simplicity and responsivity, and as potential candidates for low-cost monitoring, but PS/c-Si diodes show different optic behavior in the absorption and reflectance properties, depending on the manufacturing conditions.

In this paper we present the fabrication of macroporous silicon on crystalline silicon wafers $n$-type and $p^{+}$-type. The morphology of the macroporous layer is analyzed by SEM micrographs. The fabrication technique to produce PS surfaces is also described. In order to study the uniformity of the samples, the normalized reflectivity was measured in five zones on PS layers, in the spectral range 


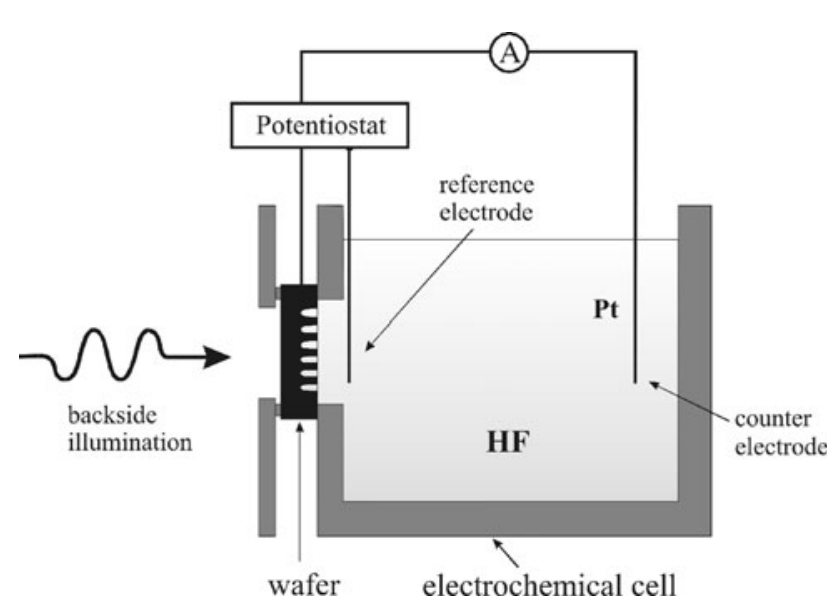

Fig. 1 Electrochemical cell used for macropores formation

of UV-Visible-NIR (200-1,100 nm) and this was compared to the typical TMAH technique of antireflective coatings on crystalline silicon.

\section{Experiments}

The basic elements for ma-PS formations with an electrochemical process are: a concentrated HF based solution as an electrolyte, and a cleaned crystalline silicon wafer. Besides, in order to obtain a good ma-PS layer, it was necessary to control the parameters of this electrochemical process [11].

Figure 1 shows the schematic electrochemical cell for macropores formation. For this formation we controlled parameters of the process as the current density and the reference voltage applied to the wafer. The samples for maPS layers were made of $n$-type and $p^{+}$-type silicon wafers with $<100>$ orientation and an average resistivity of 2-6 and $0.07-0.125 \Omega \mathrm{cm}$, respectively.

The ma-PS layer, covering one side of the wafer, was formed by means of electrochemical etching in two aqueous hydrofluoric acid (HF) concentrations: $2.5 \%$ and $15 \%$ electrolytes for $\mathrm{n}$ and $p^{+}$-types, respectively. The current density was fixed to 10 and $13 \mathrm{~mA} / \mathrm{cm}^{2}$. The etching conditions for the samples studied are summarized in Table 1. After the electrochemical HF etching, wafers were cleaned in a solution of $\mathrm{NaOH}$ for $30 \mathrm{~s}$ at $75{ }^{\circ} \mathrm{C}$.

\section{Results and discussion}

\subsection{Morphology and growth}

Figure 2a shows the SEM micrographs of the morphology of ma-PS surfaces after the electrochemical etching with
Table 1 Fabrication parameters of the samples studies

\begin{tabular}{llllll}
\hline Sample & $\mathrm{c}$-Si & $\begin{array}{l}\text { Resistivity } \\
\Omega \mathrm{cm}\end{array}$ & $\begin{array}{l}\mathrm{HF}: \mathrm{H}_{2} \mathrm{O} \\
(\%)\end{array}$ & $\begin{array}{l}\mathrm{J} \mathrm{mA} / \\
\mathrm{cm}^{2}\end{array}$ & $\begin{array}{l}\text { Etching time } \\
\mathrm{min}\end{array}$ \\
\hline A & $n$-type & $2-6$ & 2.5 & 10 & 45 \\
$\mathrm{~B}$ & $n$-type & $2-6$ & 2.5 & 10 & 60 \\
$\mathrm{C}$ & $n$-type & $2-6$ & 2.5 & 10 & 75 \\
$\mathrm{D}$ & $n$-type & $2-6$ & 2.5 & 10 & 95 \\
$\mathrm{E}$ & $p^{+}$-type & $0.075-0.125$ & 15 & 13 & 9 \\
\hline
\end{tabular}

$\mathrm{HF}$ and in Fig. $2 \mathrm{~b}$ show the cross-section of the ma-PS layers, both for $n$-type samples C $(75 \mathrm{~min}$.) and D (95 min.) and $p^{+}$-type sample $\mathrm{E}(9 \mathrm{~min}$.). Pores are randomly distributed in the surface of samples studied. For all samples, we obtained a great number of pores and thicknesses between 1 and $50 \mu \mathrm{m}$. From these views, we can evaluate the depth and the uniformity of the pores.

\subsection{Optical characterization of ma-PS ARC}

Figure 3 shows the spectral measurement setup for ARC and the different zones used in the measurement in order to study the uniformity of the samples. The monochromatic light fell with an angle of $45^{\circ}$. Each sample was studied in the wavelength range from 200 to $1,100 \mathrm{~nm}$. The normalized reflectivity coefficient of ma-PS/c-Si diodes was defined as the ratio between the reflectivity measured for each sample and the reflectivity measured from a bare silicon wafer, at a given wavelength. The reference was the same for the five samples.

Figure $4 \mathrm{a}$ shows the normalized reflectivity for the sample A (45 min of HF etching time). In this sample we obtained very small holes, because this time is the minimum time in the electrochemical process conditions for ma-PS formation. Due to this, a great dispersion in the normalized reflectivity values for the different regions was obtained (in the range of $20-55 \%$ ), indicating non-homogeneity related to different depths of the pores in the crystalline silicon. By increasing the etching time in 15 more minutes (sample B, Fig. 4b), we observed a minor dispersion in the different regions of the structure. The normalized reflectivity values were below $25 \%$. This lower reflectivity shows a better uniformity in the pores formation, as well as a greater pore depth for each measured region than in the first sample. Increasing the etching time in 15 more minutes (sample C) the porosity in the ma-PS surface was increased, which improved the light absorption. As can be seen in the Fig. $4 \mathrm{c}$, the normalized reflectivity presented the lowest values of about $10 \%$ with respect to the previous samples. Further increasing of the etching time in 20 more minutes (sample D, Fig. 4d) it increases the porosity in the ma-PS surface, which 
Fig. 2 SEM micrographs of a ma-PS surface and $\mathbf{b}$ the cross-section of the ma-PS layers for $75,95 \mathrm{~min}$ ( $n$-type) and $9 \min \left(p^{+}\right.$-type $)$
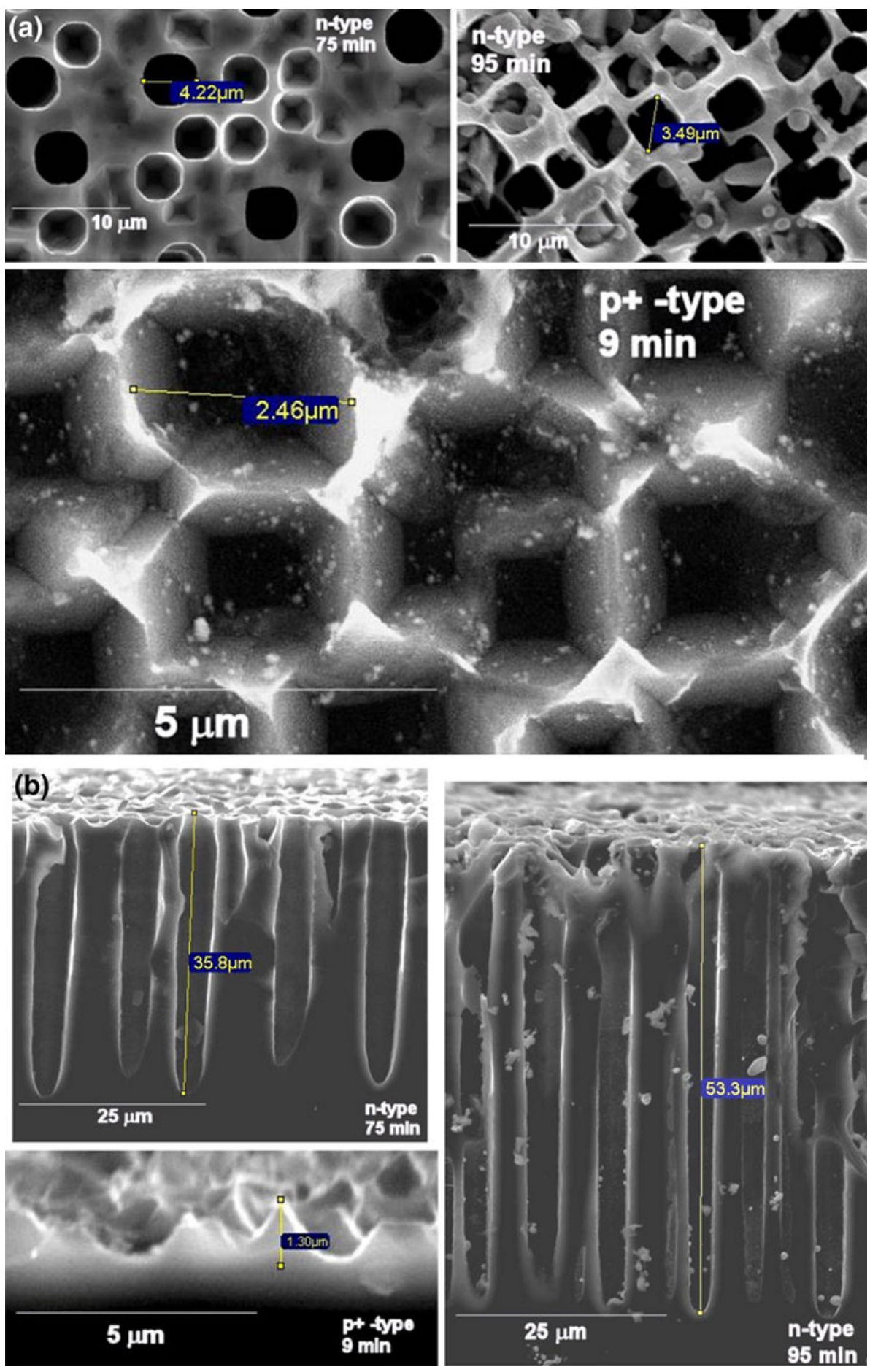

improved the light absorption considerably. The normalized reflectivity presented the lowest values of about $1 \%$ with respect to the previous samples. This seems to indicate that a critical depth exists in the formed pores. Thus, the normalized reflectivity does not vary in the different regions, due to the uniformity stabilization of the formed holes in the ma-PS/n-Si diode.
When we compared the normalized reflectivity for the samples with different substrate ( $n$-type and $p^{+}$-type), we found that the lowest value of normalized reflectivity was in the sample $\mathrm{E}\left(p^{+}\right.$-type) $<0.5 \%$, as can see in the Fig. 5. When increased the HF concentration for the $n$-type formation, we found values about $3.5 \%$ at the same etching time that sample E, but for this time the 
Fig. 3 a Spectral measurement system ARC and $\mathbf{b}$ different zones where light insides on the ma-PS surface
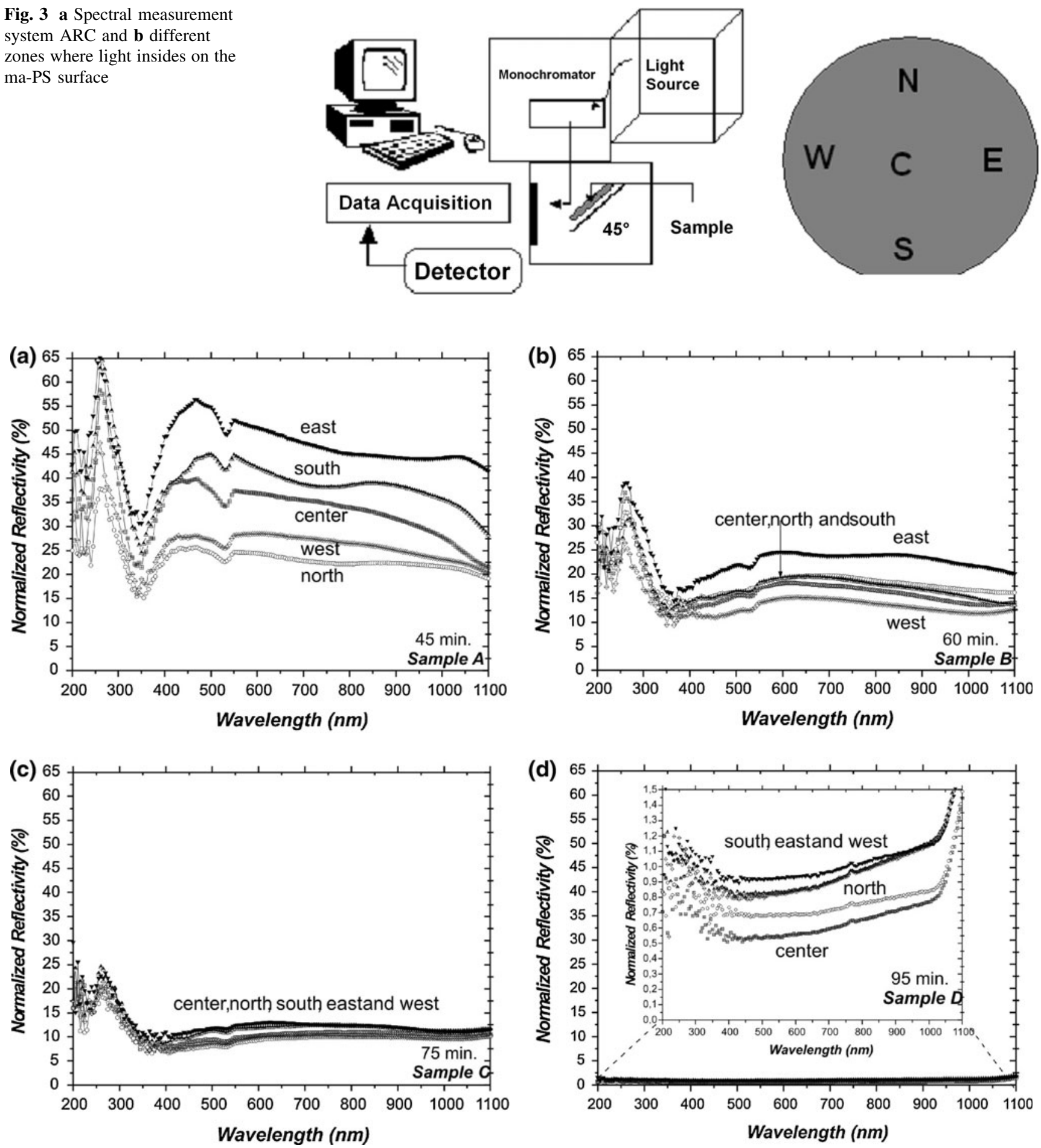

Fig. 4 Normalized reflectivity spectrum on the ma-PS/n-Si diode for etching time of 45 (a), 60 (b), 75 (c) and 95 (d) minute and an angle of $45^{\circ}$ for different zones on the wafer

layer presented a non-homogeneity related to different depths of the pores in the crystalline silicon. The problem for the sample $\mathrm{E}$ is the polish of silicon wafer, the etching is very fast for the concentration used in this sample, but is possible controlled with the current density applied to the sample.

We obtained a textured sample for TMAH technique: the experimental setup was TMAH:Iso-propane 2:1 (IPA), 


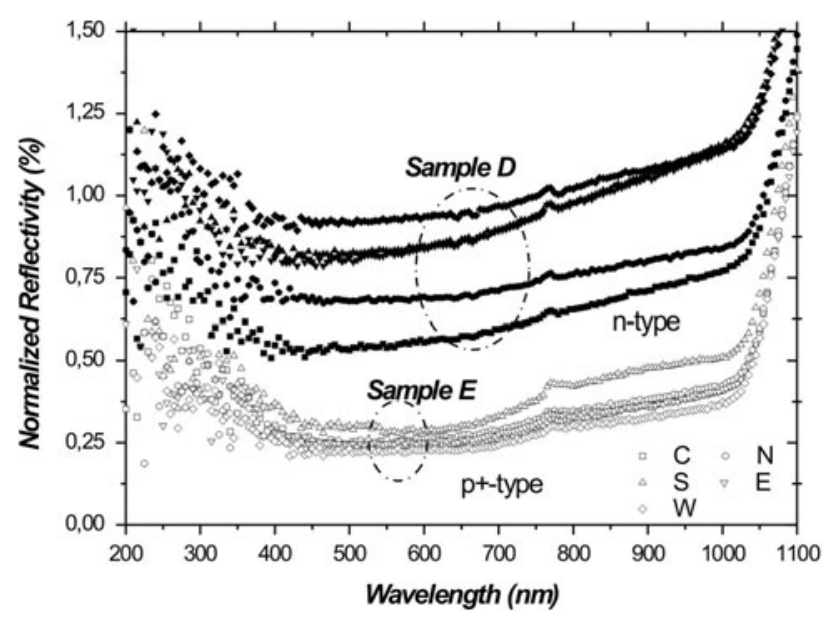

Fig. 5 Normalized reflectivity spectrum compared between the sample D $(n-\mathrm{Si})$ and sample $\mathrm{E}\left(p^{+}-\mathrm{Si}\right)$. For both samples, values below of $1 \%$, (Solid symbol ma-PS $/ n-\mathrm{Si}$ and open symbol ma-PS $/ p^{+}-\mathrm{Si}$ ) were obtained

with $80{ }^{\circ} \mathrm{C}$ of temperature and $50 \mathrm{~min}$. In the Fig. 6a, we show the SEM of the samples fabricated on $n$-Si with $10 \%$ TMAH-5\% IPA and compared with $2.5 \%$ aqueous hydrofluoric acid (HF) concentrations with $95 \mathrm{~min}$. (Sample D).
Both texturization surfaces obtained the normalized reflectivity values below of $1 \%$, as can be seen in the Fig. 6b. The result obtained for the sample TMAH was compared with the sample $\mathrm{E}$ and the normalized reflectivity was very similar about to $0.25 \%$.

\section{Conclusions}

We have developed an electrochemical process to fabricate the ma-PS layer antireflective coatings on silicon wafers. We have obtained a relationship between the HF etching time of the wafer surface and the reflectivity properties for the different samples. By increasing the etching time, the normalized reflectivity has been improved (values below $1 \%$ and lower dispersion). A critical depth exists in the formed pores, which minimizes the dispersion in the different regions for each sample, which stabilizes the uniformity and the homogeneity of the holes depth formed in the ma-PS/c-Si diode. Due to this, the value of the normalized reflectivity does not vary in the different regions of the samples.
Fig. 6 Antireflective coatings of ma-PS $/ n-\mathrm{Si}$ for different zones, a SEM micrographs of the sample compared and b normalized reflectivity compared for both texturization (Solid symbol ma-PS layer and open symbol TMAH Texturization)
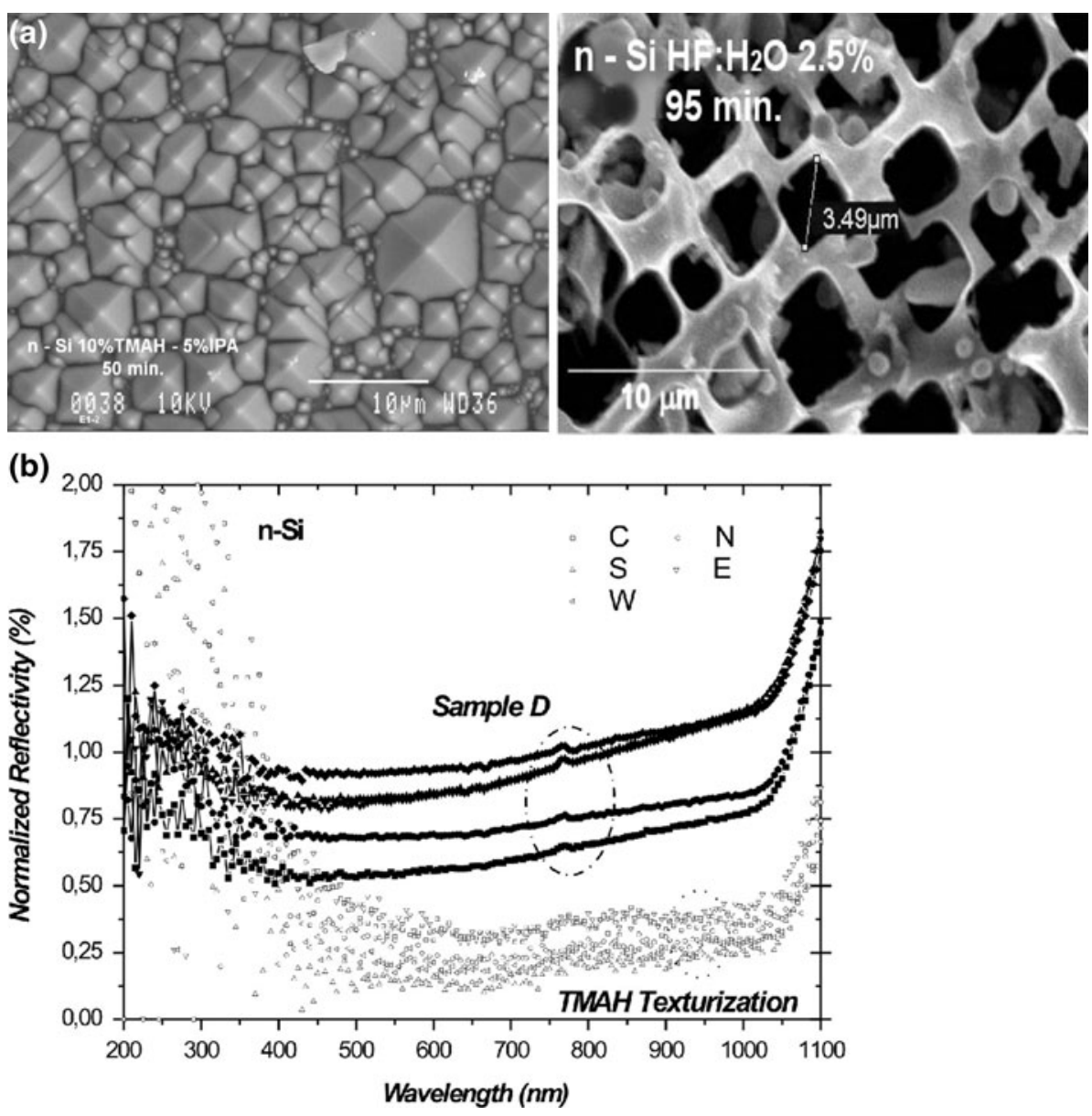
Acknowledgments The authors would like to thank Dr. J. Pallares (Universitat Rovira i Virgili) for their support in this investigation. This work was supported by the Spanish Commission of Science and Technology (CICYT) under Grant No. TIC2002-4184-C02-02 and the Universidad Autónoma de Occidente (UAO) under Project No. 07INTER-79.

\section{References}

1. A. Prasad, S. Balakrishnan, S.K. Jain, G.C. Jain, J. Electrochem. Soc. 129, 596 (1982)

2. L.T. Canham, Appl. Phys. Lett. 57, 1046 (1990)

3. S. Yae et al., Solar Ener. 80, 701 (2006)

4. B. González-Díaz et al., Physica E 38, 215 (2007)
5. T.D. James et al., in International Conferrence on Nanoscience and Nanotechnology, (ICONN 2006, Brisbane, Australia, 2006

6. M.J. Huang, C.R. Yang, Y.C. Chiou, R.T. Lee, Solar Ener. Mat. Solar Cell 92, 13526 (2008)

7. N. Marrero, R. Guerrero-Lemus, B. González-Díaz, D. Borchert, Thin Solid Film 517, 2648 (2009)

8. A.M. Rossi, H.G. Bohn, Phys. Status Solidi. (a) 202, 1644 (2005)

9. U. Gangopadhyay et al., Solar Ener. Mat. Solar Cell 91, 285 (2007)

10. F. Fonthal et al., in AIP Conferrence Proceedings VI RIAO/IX OPTILAS. vol. 992, (2008), p. 780

11. F. Fonthal, I. Torres, in 11th International Conferences on Advanced Materials, (ICAM 2009, Rio de Janeiro, Brazil 2009)

12. M.Y. Ghannam, A.A. Abouelsaood, A.S. Alomar, J. Poortmans, Solar Ener. Mat. Solar Cell 94, 850 (2010) 